\title{
Warfare Gas Detection at Trace Level Using a Multiple SAW Sensor Approach Based on Functionalized Nanodiamond Coatings
}

\author{
Benoit Tard ${ }^{1}$, Adeline Trouvé ${ }^{1}$,Emmanuel Scorsone ${ }^{1}$, Achim Voigt ${ }^{2}$, Michael Rapp ${ }^{2}$, Philippe Bergonzo $^{1}$ \\ ${ }^{1}$ CEA LIST Saclay, 91191 Gif/Yvette Cedex, \\ ${ }^{2}$ Karlsruher Institut für Technologie, Hermann-von-Helmholtz-Platz1, 76344 Eggenstein- \\ Leopoldshafen \\ Benoit.tard@cea.fr
}

\begin{abstract}
We have investigated the performances of Surface Acoustic Wave (SAW) transducers for the detection of toxic gases. SAW sensors are indeed promising because they can be operated at room temperature, offer high sensitivity and can potentially be mass-manufactured. However, the selectivity of such acoustic sensors requires the use of sensitive layers that when deposited on the transducer's surface may affect drastically the wave propagation and therefore the reliability of the sensors. Hence, strong efforts were dedicated to develop new concepts for sensitive coatings that remain very thin so as not to alter the acoustical properties of the device, very stable, and very versatile for the immobilization of selective receptors. In this scope we have developed a novel approach based on the use of Nanodiamonds (NDs).
\end{abstract}

Key words: Surface acoustic wave, toxic gases, sensitive layers, nanodiamond.

\section{Nanodiamonds coatings}

Diamond nano-particles (NDs) can be fabricated either by grinding of High Pressure High Temperature (HPHT) diamond particles or by detonation processes. They were deposited as homogeneous layers on quartz SAW resonators. The stability of the particles combined with their carbon terminated surface offers wide opportunities for surface modification through a wide range of plasma treatments or chemical grafting using various routes derived from standard organic chemistry. This surface modification enables to tailor the possible interactions of the surface with the gas species to be sensed (nerve agents, explosives). Thus the highly versatile, stable and porous nature of the NDs matrix is promising for the development of sensitive coatings for chemical detection in the gas phase, especially when combined with gravimetric transducers, such as surface acoustic wave transducers.

In this study the SAW transducers along with the SAGAS read out electronic system were provided by the Karlsruhe Institute of Technology. This multi-channel sensor system enabled us to characterise in parallel the response of 8 sensors and to focus our study on the ND coatings development.
Here NDs were deposited as thin porous nanometric coatings over the transducers surface using a process described elsewhere [1]. The thickness homogeneity of the coatings was controlled by SEM imaging and ellipsometry measurements.

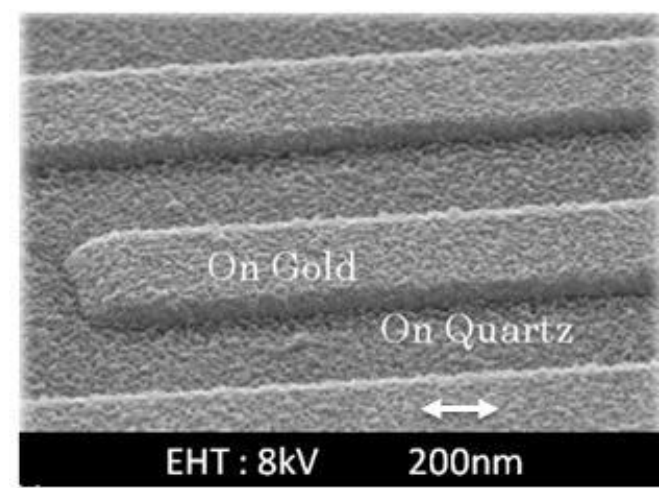

Figure 1 : SEM image of NDs coating on the IDT electrodes of a SAW transducer

\section{Nanodiamond surface termination \\ Several routes were explored for immobilizing organic receptors onto the surface of the NDs, either by electrostatic attachment [2] or C-C covalent bonding [3]. For example, a strong hydrogen-bond acidic fluorinated alcohol moiety exhibiting a strong affinity with basic}


organophosphorous compounds (nerve gases, pesticides, etc) was grafted on the ND porous layer [4]. Other surface modifications either based on wet oxidation of the surface or based on specific plasma treatments were also investigated. The success of immobilization was validated either with XPS or FTIR analysis or both.

\section{Tests under toxic gas exposures}

The resulting sensors were tested under gas exposure using a dedicated home-made gas rig. This experimental set up allows generating a range of vapours at trace levels including Dimethyl methylphosphonate (DMMP) or dinitrotoluene (DNT) as simulant for sarin gas or trinitrotoluene (TNT), respectively. In the case of sarin gas, mustard gas and phosgene, experiments were carried out using facilities at TNO (Netherland). Our gas rig allows generating e.g. DMMP from diffusion or permeation tubes to cover the range $10 \mathrm{ppb}$ to $10 \mathrm{ppm}$. Figure 2 shows a typical response of two SAW transducers with diamond coatings terminated with a fluoroalcohol moiety to 7.5 ppm DMMP at $200 \mathrm{~mL} / \mathrm{min}$ in dry nitrogen. Here a reversible response was measured with the response time $t_{90 \%}$ in the order of 40 seconds. After this typical time a plateau was observed corresponding to a frequency shift of approximately $4000 \mathrm{~Hz}$. It is worth mentioning also that for both sensors a fairly reproducible response in terms of response trends and signal intensities was observed thus suggesting the high reproducibility in the sensors preparation.

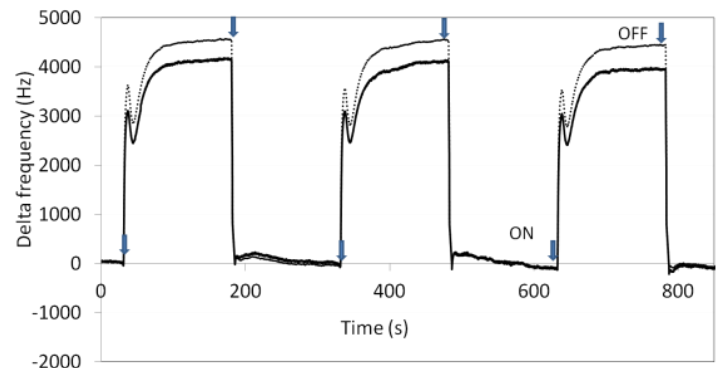

Figure 2: Transient response of two SAWs sensors with - $\left(\mathrm{CF}_{3}\right)_{2}-\mathrm{OH}$ terminated $\mathrm{ND}$ coating to thee successive exposures to $7.4 \mathrm{ppm}$ DMMP in dry nitrogen at $200 \mathrm{~mL} / \mathrm{min}$

Since the performances of the fluoroalcohol terminated sensors were promising for the detection of DMMP, the same sensors were tested against the real sarin gas for which they were expected to also exhibit a good affinity. A typical response to $2 \mathrm{ppm}$ sarin gas for two of those sensors is shown in Fig. 3.

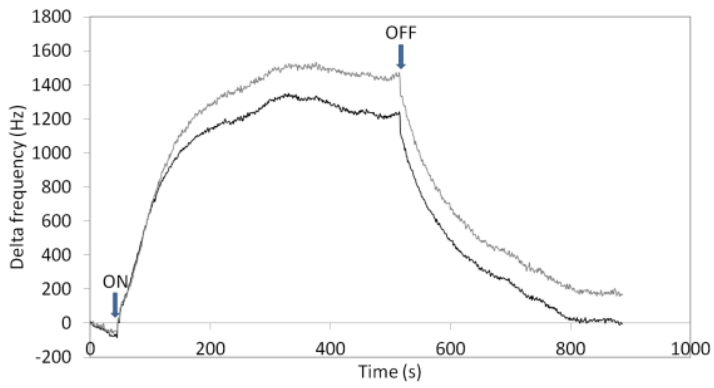

Figure 3 : Transient response of two SAWs sensors with - $\left(\mathrm{CF}_{3}\right)_{2}-\mathrm{OH}$ terminated $\mathrm{ND}$ coating to an exposure to $2 \mathrm{ppm}$ Sarin in dry nitrogene at $200 \mathrm{~mL} / \mathrm{min}$

Fig. 3 shows that a good sensitivity to sarin gas was also obtained. Moreover the responses are again reversible. The sensors signals for DMMP and for sarin gas are comparable in terms of apparent sensitivity $(750 \mathrm{~Hz}$ per ppm for Sarin gas at $2 \mathrm{ppm}, 500 \mathrm{~Hz}$ per ppm for DMMP at $7.5 \mathrm{ppm}$ ). The slightly higher sensitivity in the case of sarin gas may be explained by the difference in structural form between both analytes and the higher polarity of sarin gas over DMMP.

Highly oxidised ND coatings SAW sensors were also assessed towards the detection of mustard gas. Fig. 4 shows a typical transient response to $4 \mathrm{ppm}$ mustard gas in dry nitrogen. Here the response was very fast and so was the recovery time, both typically under 30 seconds.

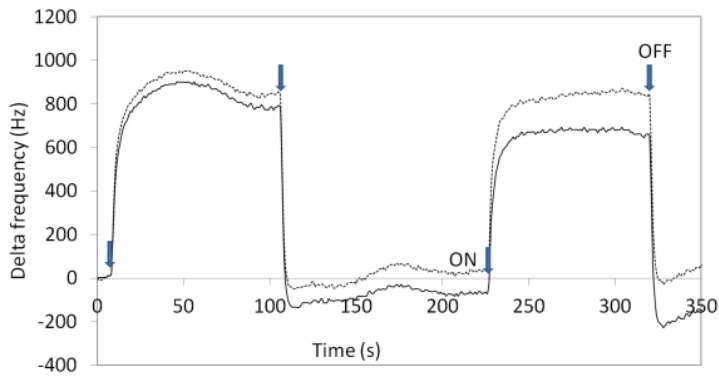

Figure 4: Transient response of two highly oxidized diamond coatings sensors to two successive exposures to mustard gas mustard gas at a concentration of $4 \mathrm{ppm}$ in dry nitrogen at $200 \mathrm{~mL} / \mathrm{min}$

The same sensors were exposed to several concentrations of mustard gas and the steady state response was used to plot the calibration curve as shown in Fig. 5. This graph shows that in the range $500 \mathrm{ppb}$ to $8 \mathrm{ppm}$ the response seems to be linear with linear regression $R^{2}$ value of 0.9973 . The sensitivity was in the order 
of $250 \mathrm{~Hz} / \mathrm{ppm}$ and the detection threshold assessed to be around $300 \mathrm{ppb}$.

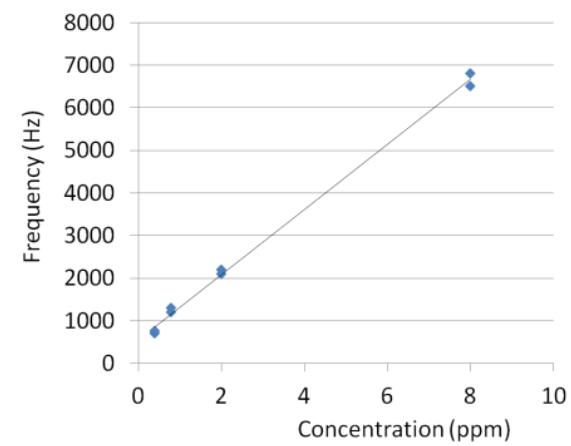

Figure 5: Calibration curve for highly oxidized ND coating SAW sensor from 500 ppb to 8 ppm mustard gas concentration in dry nitrogen

The same highly oxidized ND coatings SAW sensors were also assessed for phosgene detection. The results are shown in Fig. 5 and Fig. 6 where the sensors were exposed to 100 $\mathrm{ppb}$ and $1 \mathrm{ppm}$, respectively. For phosgene exposures, the sensors were also responding fast and the response was also fully reversible. Clearly here the detection threshold is well below 100 ppb. Again it can be observed that both sensors are very reproducible in terms of sensor intensity.

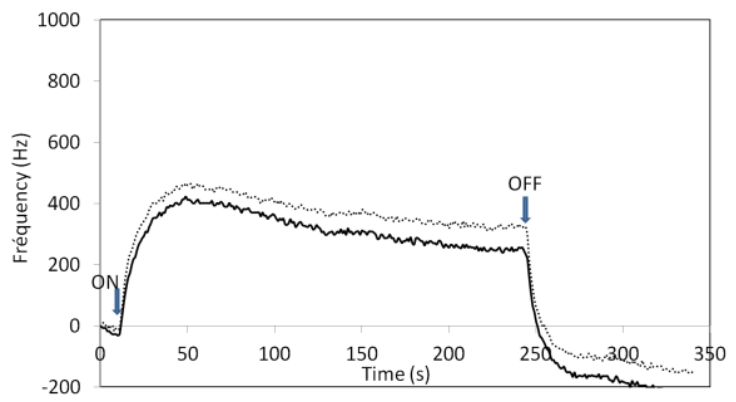

Figure 4: Transient response of two highly oxidized diamond coatings sensors to two successive exposures to phosgene at a concentration of 100ppb in dry nitrogen at $200 \mathrm{~mL} / \mathrm{min}$

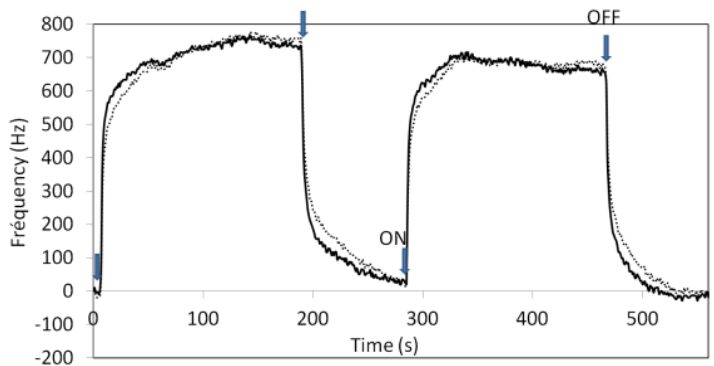

Figure 5: Transient response of two highly oxidized diamond coatings sensors to two successive exposures to phosgene at a concentration of $1 \mathrm{ppm}$ in dry nitrogen at $200 \mathrm{~mL} / \mathrm{min}$

\section{Discussion}

In this work SAW transducers were coated with ND coatings surface terminated with polar acid groups (either from surface oxidation or organic grafting strategies). Thus all those sensors feature high affinity with the toxic gases tested, namely DMMP, sarin gas, mustard gas or phosgene, via strong hydrogen bond affinity towards these target molecules. For all sensors the response is very fast and reversible. Indeed, since diamond is a very dense material, there is no diffusion possible into the coating and the sensor responses are not limited by diffusion into the sensitive coating. Instead the responses are mainly driven by surface interactions between the NDs and the target analyte. Interestingly, although more experiments would be necessary in particular to extract the true sensitivity of the sensors to the different analytes, the response of the fluoroalcohol terminated sensors seems to be comparable in terms of response time and reactivity towards the simulant DMMP and the real sarin gas. Even though the chemical composition of both analytes is similar sarin has actually a significantly lower boiling point than DMMP and is more polar. This might explain the slightly higher sensitivity of the sensor to sarin gas with respect to DMMP. More generally it appears that the sensitivity seems to increase with decreasing boiling points. Clearly those sensors are not selective to the toxic gases under investigation. Nevertheless they are highly promising for the design of multisensor arrays. The sensors are highly repeatable and reproducible, which is a crucial asset when selectivity is performed using data training and pattern recognition.

\section{Conclusion and perspectives}

In this study, SAW transducers coated with modified ND nanolayers were investigated for the detection of warfare gases. A reproducible process was found to deposit ND layers which are thin enough not to degrade the acoustic properties of the resonators, and uniform in thickness. This $\mathrm{sp}^{3}$ carbon sensitive interface can be terminated with a wide range of chemical groups and by several routes (plasma, organic grating, etc) allowing to tune the affinity of the surface with target analytes. Examples of sensors were shown here for the detection of warefare gases such as sarin gas, mustard gas and phosgene. High sensitivities were achieved as a result of the combination of highly sensitive gravimetric SAW transducers and high affinity ND coatings. Fast response times well below the minute were also observed and explained by the nature of the sensitive coatings. 
Altogether those results and the large versatility and stability of the ND coatings are very promising towards the development of multisensor arrays for gas detection and identification.

\section{Acknowledgements}

Partners from TNO are acknowledged to have enabled those measurements in best conditions.

\section{References}

[1] Girard, H. A., Perruchas, S., Gesset, C., Chaigneau, M., Vieille, L., Arnault, J.-C., Bergonzo, P., et al. (2009). Electrostatic Grafting of Diamond Nanoparticles: A Versatile Route to Nanocrystalline Diamond Thin Films. ACS Applied Materials \& Interfaces, 1(12), 2738-2746. doi:10.1021/am900458g

[2] Chevallier, E., Scorsone, E., Girard, H. a, Pichot, V., Spitzer, D., \& Bergonzo, P. (2010). Metalloporphyrin-functionalised diamond nanoparticles as sensitive layer for nitroaromatic vapours detection at room-temperature. Sensors and Actuators B: Chemical, 151(1), 191-197. doi:10.1016/j.snb.2010.09.022

[3] Girard, H. A., Arnault, J. C., Perruchas, S., Saada, S., Gacoin, T., Boilot, J. P., \& Bergonzo, P. (2010). Hydrogenation of nanodiamonds using MPCVD: A new route toward organic functionalization. Diamond \& Related Materials, 19(7-9),1117-1123.doi:10.1016/ j.diamond.2010. 03.019

[4] Qi Zheng, Yong-chun Fu, Jia-qiang Xu, Advances in the chemical sensors for the detection of DMMP - A simulant for nerve agent sarin, Procedia Engineering, Volume 7, 2010, Pages 179-184, ISSN 1877-7058, 10.1016/j.proeng.2010.11.027. 